\title{
Clinical outcome scoring of intra-articular calcaneal
}

\section{fractures}

Tim Schepers MD ${ }^{1}$, Martin J. Heetveld MD PhD², Paul G.H. Mulder $\mathrm{PhD}^{3}$, Peter Patka MD $\mathrm{PhD}^{1}$

${ }^{1)}$ Erasmus MC, University Medical Center Rotterdam, Department of SurgeryTraumatology, the Netherlands.

${ }^{2)}$ Kennemer Gasthuis, Dept. of Surgery, P.O. Box 417, 2000 AK Haarlem, the Netherlands

${ }^{3)}$ Erasmus University Rotterdam, Department of Epidemiology \& Biostatistics, the Netherlands

No conflicts of interest are stated by any of the authors.

Approval of the Ethical Board Review was obtained prior to this study.

Corresponding author:

T. Schepers, MD

Erasmus MC, University Medical Center Rotterdam

Department of Surgery-Traumatology

Room H9-74

P.O. Box 2040

3000 CA Rotterdam, the Netherlands

E-mail: t.schepers@erasmusmc.nl

Phone: +31-10-4631050

Fax: +31-10-4632396 


\begin{abstract}
Introduction: Outcome reporting of intra-articular calcaneal fractures is inconsistent. This study aimed to identify the most cited outcome scores in the literature and to analyse their reliability and validity.

Method: A systematic literature search identified 34 different outcome scores. The most cited outcome score was the American Orthopaedic Foot and Ankle Society Hindfoot (AOFAS) score, followed by the Maryland Foot Score (MFS) and the Creighton-Nebraska score $(\mathrm{CN})$. Reliability (internal consistency) and validity (content, construct, and criterion) were determined for the three outcome scoring systems.

Results: Internal consistency (Cronbach's alpha, reliability) was similar for the MFS $(\alpha=0.82)$ and AOFAS $(\alpha=0.78)$, but lower for the CN $(\alpha=0.61)$. Floor- and ceiling-effects were good for all three scores. The individual items within these outcome scores showing best content validity were pain, return to work, subtalar range of motion, walking distance, ankle range of motion, and gait abnormalities or limping. Construct validity was good for all individual items except sagittal motion, stability at physical exam, and shoe size. All three outcome scores showed high correlation with patient satisfaction as measured with a Visual Analogue Scale (VAS, criterion validity) and indication for an arthrodesis.

Conclusions: Pending consensus we would recommend choosing between the widely accepted, reliable and valid AOFAS Hindfoot and the Maryland Foot Score as the scoring system of choice.
\end{abstract}

Keywords: Calcaneus, fracture, intra-articular, outcome scoring. 


\section{Introduction}

Outcome reporting in randomised controlled trials and meta-analysis of intra-articular calcaneal fractures is inconsistent. Various outcome scores are applied by different research groups. ${ }^{1-3}$ Consensus on one outcome scoring system for the assessment of outcome in displaced intra-articular calcaneal fractures would aid in the comparison of results of multiple studies with comparable methodologies, and combining different smaller prospective trials into a meta-analysis. ${ }^{45}$

Patient-based outcome scores can be divided into two groups. The first group consists of the generic instruments such as the Short Form 36, Visual Analogue Scales and other quality-of-life scores, which look at a wide variety of general health issues. ${ }^{6}$ ${ }^{7}$ The second group consists of disease-specific instruments, which focus on patient perception of one specific condition, for example calcaneal fractures. ${ }^{6}$

Prior to implementation scoring systems should be tested for reliability (e.g.: internal consistency, test-retest, intra-observer and inter-observer agreement), validity (e.g.: content, construct, and criterion), and responsiveness. ${ }^{89}$ Few scoring systems in foot and ankle surgery have been tested for reliability and validity after they were developed. ${ }^{9}$ The aim of this study was first to identify widely accepted outcome scores used in intra-articular calcaneal fractures in the literature and their individual items. The reliability (internal consistency) and validity (content, construct, and criterion) of three most cited outcome scoring systems was then determined in a cohort of patients with a displaced intra-articular calcaneal fracture. 


\section{Patients and methods}

\section{Literature search}

A literature search was conducted in the electronic databases of Embase, Cochrane Library and Pubmed using the following search-terms and Boolean operators: 'calcaneus' OR 'os calcis' OR 'calcaneum' OR 'calcaneal' AND 'fracture' up to December 2006. Articles were requested at the university medical (internet) library and were reviewed by two authors (TS and MH). An article was found eligible when it concerned the treatment of patients with intra-articular calcaneal fractures. Additionally a comprehensive search of reference lists of published articles and review articles was conducted to find additional studies. The applied outcome scoring systems were extracted from all these articles. In determining reliability and validity we restricted the next analysis to the three most frequently cited widely accepted outcome scores.

\section{Patients}

After exclusion of patients ( 25 percent) who had demised $(n=2)$, were emigrated $(n=4)$, had an unknown address $(n=5)$ or already had a secondary arthrodesis performed $(n=5)$, a total of 48 patients with 59 displaced intra-articular fractures of the posterior facet of the calcaneus were surgically treated in our level-1 traumacenter from 1999 to 2004. Patients who responded for clinical interview and examination, after IRB agreement and informed consent were given, had a mean follow-up of 34 months (range 13 to 75 months). The median age was 49 (SD 13) years at trauma, $72 \%$ were male. According to the Sanders classification ${ }^{10}$ there were 23 type II, 18 type III and 18 type IV fractures. The percutaneous reduction and fixation, modified from Forgon and Zadravecz ${ }^{11}$, was the only surgical approach for displaced intra- 
articular calcaneal fractures used in our hospital. This technique relies on the principle of a triangular distracting force between the talar neck, the calcaneal tuber and the cuboid, followed by percutaneous screw fixation.

All patients were informed about the current study and asked to complete the American Orthopaedic Foot and Ankle Society Hindfoot score (AOFAS), the Maryland Foot Score (MFS) and the Creighton-Nebraska score $(\mathrm{CN})$, which each have a range of $0-100$ points. Patients with a bilateral fracture were instructed to report on the side with the least satisfactory result. ${ }^{11}$

In addition to the three different outcome scores, patient satisfaction with the overall outcome was assessed with a single-question Visual Analogue Scale (VAS) analysed on a scale of 100 millimetres $(\mathrm{mm}) .{ }^{12}$ Zero $\mathrm{mm}$ represented no satisfaction at all and $100 \mathrm{~mm}$ was scored if patient satisfaction was excellent. ${ }^{12}$ There is no consensus or gold-standard scoring system in foot and ankle surgery to date. Correlations between foot-ankle related outcome scores and the more generic health Medical Outcome Study Short Form-36 show moderate coefficients. ${ }^{13}{ }^{14}$. We therefore chose to use the single-question Visual Analogue Scale (VAS) to measure overall patient satisfaction with treatment result, which has been shown a moderate correlation with the SF-36 and a good correlation with the disease-specific outcome (Rowe) score in patients with a calcaneal fracture. ${ }^{12} 15$

\section{Statistical analysis}

The statistical analysis was performed using the Statistical Package for the Social Sciences (SPSS) version 12.0 (SPSS, Chicago, IL, USA). Correlations were considered statistically significant with a p-value of $<0.05$. 


\section{-Reliability}

To determine reliability of the three outcome scoring systems the internal consistency as represented by the Cronbach's alpha, was calculated. ${ }^{8}$ The Cronbach's alpha indicates the extent to which a set of test items measure a single variable like outcome. A Cronbach's alpha of 0.70 to 0.80 is regarded as satisfactory. For clinical application values up to 0.95 are desirable. ${ }^{16}$

Another aspect of reliability is whether the scoring system measures the full range of the disease or complaints. ${ }^{17}$ The incidence of minimum (floor; zero points) and maximum (ceiling; 100 points) scores was calculated for all used outcome scores. A score with low floor and ceiling effect, below 10 percent, can differentiate better between patients at the high and low end of the outcome scoring system. ${ }^{17}$

\section{-Validity}

Content validity determines if the domain of interest is comprehensively sampled by the items, or questions, in the instrument (i.e. scoring system). ${ }^{18}$ Two means of determining content validity exist: piloting the instrument with representative respondents or item selection by expert panels. ${ }^{19}$ Therefore the outcome scores were broken down into their individual items to determine the frequency with which these items were used. These individual items of available outcome-scores in the literature were selected by expert panels. The most frequently used items are expected to have the largest content validity. The following four items, determined at physical examination, were added: the range of motion of the ankle and subtalar joint, heel width (in mm) measured from the plantar side at the level of both malleoli, calf circumflex $15 \mathrm{~cm}$ beneath the knee joint (in $\mathrm{mm}$ ) and the ability to walk on heels and toes were measured (yes/no). 
Construct validity shows whether the items and outcome score measure what they should measure. All individual scoring items of the three most frequently used outcome scores and additional frequently used individual items were correlated with the total result of the three scoring systems and the VAS. The Spearman rank correlation coefficient was used for this 'item versus total score correlation'.

Criterion validity is the correlation between the developed score and an accepted validated score. ${ }^{17}$ The total scores of the scoring systems were correlated with patient satisfaction (VAS) as a measure of criterion validity. The 'indication for an arthrodesis' was used as an alternative measure of criterion validity. This indication was defined as a persisting, deteriorating limitation in daily activities due to pain at the level of the subtalar joint, with a positive, pain relieving effect of a Lidocain ${ }^{\circledR}$ injection into the subtalar joint. ${ }^{20} 21$ Patients who had already undergone a secondary arthrodesis were deliberately excluded prior to the study, because of the low reliability of retrospective assessment of the clinical status before the secondary arthrodesis with an outcome scoring system. ${ }^{22}$

The odds ratio per unit of the total score with $95 \%$ confidence interval (CI) was calculated for the dichotomous outcome variable indication for an arthrodesis (yes/no response)'. This correlation gives information on the predictive value of the entire score in predicting the secondary arthrodesis.

The Spearman rank correlation coefficient was used for testing relationships between the VAS and the total score of the three scoring systems and also for the correlation between the three scoring systems. To determine paired differences between the three most cited scoring systems the Wilcoxon signed rank test was used. 


\section{Results}

\section{Literature search}

In 195 studies (30\%) a total of 34 outcome scoring systems were identified (Table 1). The three most frequently cited outcome scores were the American Orthopaedic Foot and Ankle Society Hindfoot score (AOFAS), ${ }^{23}$ the Maryland Foot Score (MFS) ${ }^{10}$ and the Creighton-Nebraska score $(\mathrm{CN})^{24}$. A total of 49 different subjective and physical exam items were extracted from 26 outcome scores; a median of 6.5 items per outcome scoring system.

\section{Reliability}

The Cronbach's alpha coefficient, as measure of the internal consistency, was 0.78 for the AOFAS, 0.82 for the MFS and 0.61 for the $\mathrm{CN}$ score.

The ceiling score was encountered in $8 \%$ for the AOFAS and the $\mathrm{CN}, 0 \%$ for the MFS and $13 \%$ for the VAS. The floor effect was seen in $0 \%$ for the diseasespecific scores and in $2 \%$ for the VAS.

\section{Validity}

Six items showed good content validity. These items (Figure 1) were pain (encountered in 21 scoring systems), return to work $(n=13)$, subtalar range of motion $(\mathrm{n}=13)$, walking distance $(\mathrm{n}=12)$, ankle range of motion $(\mathrm{n}=10)$, and gait abnormalities or limping $(n=10)$. Five out of these six items are used in the AOFAS and MFS score, and four in the $\mathrm{CN}$ score.

A total of 16 items showed construct validity by correlating with all outcome scores and with the VAS. The AOFAS and the MFS score correlated significantly with 16 (70\%) items, the $\mathrm{CN}$ with a total of $17(74 \%)$ items, and the VAS with $16(70 \%)$ items (table 2). Items showing no correlation with any of the outcome scores were sagittal 
motion (ankle ROM), stability at physical exam, shoe size, walking on heels, heel width and calf diameter.

There was a significant correlation between the total-scores with the Spearman rank test: between MFS and AOFAS $(r=0.84, \mathrm{p}<0.001)$, between CN and AOFAS $(r=$ 0.84, $\mathrm{p}<0.001)$ and between MFS and CN $(\mathrm{r}=0.79, \mathrm{p}<0.001)$. The Wilcoxon signed ranks test showed a significantly better outcome measured with the AOFAS score than the MFS $(\mathrm{p}<0.001)$ and the CN $(\mathrm{p}<0.001)$ scores. The MFS resulted in significantly better outcome than the $\mathrm{CN}$ score $(\mathrm{p}<0.027)$ per patient.

As a measure of criterion validity, all total outcome scores correlated significantly with overall patient satisfaction as measured on the Visual Analogue Scale (AOFAS r $=0.75$, MFS $\mathrm{r}=0.72, \mathrm{CN} \mathrm{r}=0.76, \mathrm{p}<0.001$ in all cases). All total-scores also correlated significantly with the indication for an arthrodesis (odds ratio AOFAS = 0.93 (CI: 0.87-0.99), $\mathrm{p}=0.02$; odds ratio MFS $=0.94$ (CI: 0.89-0.99), $\mathrm{p}=0.02$; odds ratio $\mathrm{CN}=0.94(\mathrm{CI}: 0.89-0.99), \mathrm{p}=0.03)$. 


\section{Discussion}

The literature search performed in this study showed a large amount of and variation in existing calcaneal fracture outcome scoring systems. No outcome scoring system was applied in approximately $70 \%$ of the articles. The AOFAS was cited most frequently and appears to be the most widely accepted scoring system. Even though citation frequency does not necessarily reflect clinical relevance, broad acceptance of a single outcome score would facilitate comparison of multiple studies. ${ }^{25}$

Our search represents a first comprehensive analysis of outcome scoring systems specifically used in determining outcome in calcaneal fractures in the literature, identifying thirty-four outcome scores. A restricted meta-analysis on outcome rating scales in general foot and ankle surgery showed forty-nine scoring systems, of which eighteen were cited more than once. ${ }^{9}$ The AOFAS hindfoot score was also the most frequently applied. No scoring system in the current literature was identified as being reliable, valid or responsive. ${ }^{9}$ An earlier attempt to identify a rational scoring system combined the four most important individual items (pain at rest, pain at activity, return to work, ambulation and walking aids) of six outcome scores (Rowe, CN, Buckley, March, Paley, MFS) into a multiple regression model and found that their Kerr-Atkins score correlated equally good with outcome with only four items, suggesting that not all scoring items contribute equally in predicting outcome. ${ }^{26}$

The internal consistency (reliability) of the AOFAS and the MFS were similarly satisfactory and both scoring systems correlated well with individual subjective and objective patient outcome characteristics. The $\mathrm{CN}$ score showed lower internal consistency and appears less suitable as outcome score. All three scores showed good floor and ceiling effects (reliability). The individual items showing best content validity were pain, return to work, subtalar range of motion, walking distance, ankle 
range of motion, and gait abnormalities or limping. Most individual items correlated with outcome (construct validity). The AOFAS, MFS and CN scoring systems had similar good correlations with patient satisfaction (VAS) and the indication for an arthrodesis (criterion validity). In terms of test-retest reliability, previous research has shown good intra- and interobserver agreement of the AOFAS Hindfoot score. ${ }^{27}$

Per patient the total AOFAS scored significantly higher than the MFS, which scored significantly higher than the $\mathrm{CN}$. This is due to the different weighting of the outcome score items per scoring system. Patients frequently scored low on the important CN item 'return to work', which was more heavily weighted in the total $\mathrm{CN}$ score compared to the AOFAS item 'activity limitations'. The AOFAS could therefore underrate the value of returning to a previous level of activity or occupation. In contrast the internal consistency of the $\mathrm{CN}$ was lower than the internal consistency of the AOFAS and the MFS.

Other studies have compared the SF-36 with the AOFAS showing moderate correlations, indicating that a good disease-specific status does not always correlate with good generic health. ${ }^{13} 14$ Therefore the SF-36 might not be the proper score to compare disease-specific scores for calcaneal fractures with.

Hildebrand et al. found a significant association between scoring systems and patient satisfaction (VAS) with the overall result. The VAS as designed by Hildebrand to determine overall result (satisfaction), showed significant correlation with the SF-36 and the disease-specific outcome score by Rowe with good correlation coefficients ( 0.7 and 0.8 respectively), in patients treated by open reduction and internal fixation versus patients treated conservatively. ${ }^{12}$ The good correlation between the outcome scoring systems and the indication for an arthrodesis suggests that 'the indication for an arthrodesis' is a valid clinical endpoint. 
In this study the calculations for the three most frequently applied outcome scoring systems were performed for a cohort of patients that were all treated with the same treatment modality, i.e. by percutaneous reduction and fixation. This way a more homogeneous group was established. Although this might be considered a limitation, there are no indications that the operative technique used had affected the reliability and validity of the used outcome scoring systems. Both the ceiling and the floor effects showed the utilization of the entire range of the outcome scores, indicating that all levels of outcome were included in the study.

In summary, the American Orthopaedic Foot and Ankle Society Hindfoot Score is the most applied and cited score out of 34 outcome scoring systems used in calcaneal fractures, followed by the Maryland Foot Score and the Creighton-Nebraska score. Reliability as measured by the internal consistency was good for the AOFAS and the MFS. All three scores measured the full range of complaints. Six out of 23 individual items showed good content validity and 16 items showed good construct validity. During consensus discussion for clinical outcome reporting of intra-articular calcaneal fractures, we would recommend choosing between the widely accepted, reliable and valid AOFAS Hindfoot and the Maryland Foot Score as the scoring systems of choice. 


\section{References}

1. Bajammal S, Tornetta P, 3rd, Sanders D, Bhandari M. Displaced intra-articular calcaneal fractures. J Orthop Trauma 19:360-364, 2005

2. Bridgman SA, Dunn KM, McBride DJ, Richards PJ. Interventions for treating calcaneal fractures. Cochrane Database Syst Rev:CD001161, 2000.

3. Geel CW, Flemister AS, Jr. Standardized treatment of intra-articular calcaneal fractures using an oblique lateral incision and no bone graft. J Trauma 50:1083-1089, 2001.

4. Follak N, Merk M. The benefit of gait analysis in functional diagnostics in the rehabilitation in patients after operative treatment of calcaneal fractures. Foot Ankle Surg 9:209-214, 2003.

5. Parker M. Sample size and statistical power of randomised, controlled trials in orthopaedics. J Bone Joint Surg Br 83:1210, 2001

6. Dawson J, Carr A. Outcomes evaluation in orthopaedics. J Bone Joint Surg Br 83:313-315, 2001.

7. Ware JE, Jr., Sherbourne CD. The MOS 36-item short-form health survey (SF-36). I. Conceptual framework and item selection. Med Care 30:473-483, 1992.

8. Cronbach LJ, Meehl PE. Construct validity in psychological tests. Psychol Bull 52:281-302, 1955.

9. Button G, Pinney S. A meta-analysis of outcome rating scales in foot and ankle surgery: is there a valid, reliable, and responsive system? Foot Ankle Int 25:521-525, 2004

10. Sanders R, Fortin P, DiPasquale T, Walling A. Operative treatment in 120 displaced intraarticular calcaneal fractures. Results using a prognostic computed tomography scan classification. Clin Orthop:87-95, 1993.

11. Zadravecz G, Szekeres P. [Late results of our treatment method in calcaneus fractures]. Aktuelle Traumatol $14: 218-226,1984$

12. Hildebrand KA, Buckley RE, Mohtadi NG, Faris P. Functional outcome measures after displaced intraarticular calcaneal fractures. J Bone Joint Surg Br 78:119-123, 1996.

13. SooHoo NF, Shuler M, Fleming LL. Evaluation of the validity of the AOFAS Clinical Rating Systems by correlation to the SF-36. Foot Ankle Int 24:50-55, 2003.

14. Westphal T, Piatek S, Halm JP, Schubert S, Winckler S. Outcome of surgically treated intraarticular calcaneus fractures--SF-36 compared with AOFAS and MFS. Acta Orthop Scand 75:750-755, 2004.

15. Rowe C, Sakellarides H, Freeman P, Sorbie C. Fractures of the os calcis. JAMA 184:98-101, 1963.

16. Bland JM, Altman DG. Cronbach's alpha. Bmj 314:572, 1997.

17. Beaton DE, Richards RR. Measuring function of the shoulder. A cross-sectional comparison of five questionnaires. J Bone Joint Surg Am 78:882-890, 1996.

18. Guyatt GH, Feeny DH, Patrick DL. Measuring health-related quality of life. Ann Intern Med 118:622-629, 1993. 
19. Bot SD, Terwee CB, van der Windt DA, Bouter LM, Dekker J, de Vet HC. Clinimetric evaluation of shoulder disability questionnaires: a systematic review of the literature. Ann Rheum Dis 63:335-341, 2004.

20. Myerson M, Quill GE, Jr. Late complications of fractures of the calcaneus. J Bone Joint Surg Am 75:331-341, 1993.

21. Thomas FB. Arthrodesis of the subtalar joint. J Bone Joint Surg Br 49:93-97, 1967.

22. Toolan BC, Wright Quinones VJ, Cunningham BJ, Brage ME. An evaluation of the use of retrospectively acquired preoperative AOFAS clinical rating scores to assess surgical outcome after elective foot and ankle surgery. Foot Ankle Int 22:775-778, 2001.

23. Kitaoka HB, Alexander IJ, Adelaar RS, Nunley JA, Myerson MS, Sanders M. Clinical rating systems for the ankle-hindfoot, midfoot, hallux, and lesser toes. Foot Ankle Int 15:349-353, 1994.

24. Crosby LA, Fitzgibbons T. Computerized tomography scanning of acute intra-articular fractures of the calcaneus. A new classification system. J Bone Joint Surg Am 72:852-859, 1990.

25. Thermann H, Tscherne H. [Therapy for intraarticular calcaneal fractures]. Unfallchirurg 102:151, 1999.

26. Kerr PS, Prothero DL, Atkins RM. Assessing outcome following calcaneal fracture: a rational scoring system. Injury 27:35-38, 1996.

27. Niki H, Aoki H, Inokuchi S, Ozeki S, Kinoshita M, Kura H, Tanaka Y, Noguchi M, Nomura S, Hatori M, Tatsunami S. Development and reliability of a standard rating system for outcome measurement of foot and ankle disorders II: interclinician and intraclinician reliability and validity of the newly established standard rating scales and Japanese Orthopaedic Association rating scale. J Orthop Sci 10:466-474, 2005.

28. Allan JH. The open reduction of fractures of the os calcis. Ann Surg 141:890-900, 1955.

29. Maxfield JE, McDermott F. Experiences with the Palmer open reduction of fractures of the calcaneus. J Bone Joint Surg Am 37-A:99-106, 1955.

30. Lindsay WR, Dewar FP. Fractures of the os calcis. Am J Surg 95:555-576, 1958.

31. Nade S, Monahan PR. Fractures of the calcaneum: a study of the long-term prognosis. Injury 4:200-207, 1973.

32. Fayt P. [Evaluation of functional results after fractures of the calcaneus (author's transl)]. Rev Chir Orthop Reparatrice Appar Mot 64:661-665, 1978.

33. Forgon M, Zadravecz G. Die Kalkaneusfraktur. Springer-Verlag. Berlin, 1990:1-104.

34. Noble J, McQuillan WM. Early posterior subtalar fusion in the treatment of fractures of the os calcis. J Bone Joint Surg Br 61:90-93, 1979.

35. Hammel E, Berard P. [Calcaneal fractures]. Med Chir Pied 20:103-109, 2004.

36. Dubois D, Revuelta N, Blatt JL, Maynou C, Migaud H, Thevenon A. [Tridimensional gait analysis after unilateral subtalar arthrodesis]. Rev Chir Orthop Reparatrice Appar Mot 87:685-695, 2001.

37. Zwipp H, Tscherne H, Wulker N, Grote R. [Intra-articular fracture of the calcaneus. Classification, assessment and surgical procedures]. Unfallchirurg 92:117-129, 1989. 
38. Bradley SA, Davies AM. Computed tomographic assessment of old calcaneal fractures. Br J Radiol 63:926933, 1990.

39. Melcher G, Bereiter H, Leutenegger A, Ruedi T. Results of operative treatment for intra-articular fractures of the calcaneus. J Trauma 31:234-238, 1991.

40. Buckley RE, Meek RN. Comparison of open versus closed reduction of intraarticular calcaneal fractures: a matched cohort in workmen. J Orthop Trauma 6:216-222, 1992.

41. Parmar HV, Triffitt PD, Gregg PJ. Intra-articular fractures of the calcaneum treated operatively or conservatively. A prospective study. J Bone Joint Surg Br 75:932-937, 1993.

42. Paley D, Hall H. Intra-articular fractures of the calcaneus. A critical analysis of results and prognostic factors. J Bone Joint Surg Am 75:342-354, 1993.

43. Letournel E. Open treatment of acute calcaneal fractures. Clin Orthop:60-67, 1993.

44. Fernandez DL, Koella C. Combined percutaneous and "minimal" internal fixation for displaced articular fractures of the calcaneus. Clin Orthop:108-116, 1993.

45. Kundel K, Funk E, Brutscher M, Bickel R. Calcaneal fractures: operative versus nonoperative treatment. J Trauma 41:839-845, 1996.

46. Thordarson DB, Krieger LE. Operative vs. nonoperative treatment of intra-articular fractures of the calcaneus: a prospective randomized trial. Foot Ankle Int 17:2-9, 1996.

47. Herscovici D, Jr., Widmaier J, Scaduto JM, Sanders RW, Walling A. Operative treatment of calcaneal fractures in elderly patients. J Bone Joint Surg Am 87:1260-1264, 2005.

48. Morin P, Buckley R, Stewart R, Vande Gutche R. Oral analogue scale as an outcome measure after displaced intra-articular calcaneal fractures. Foot Ankle Int 19:694-697, 1998.

49. Stromsoe K, Mork E, Hem ES. Open reduction and internal fixation in 46 displaced intraarticular calcaneal fractures. Injury 29:313-316, 1998.

50. Thermann H, Hufner T, Schratt HE, Albrecht K, Tscherne H. [Treatment of intraarticular calcaneal fractures in adults. A treatment algorithm]. Unfallchirurg 102:152-166, 1999.

51. Schwall R, Junge RH, Zenker W, Besch L. [Treatment of intra-articular calcaneus fractures with a paraarticular external fixator]]. Unfallchirurg 103:1065-1072, 2000. 


\section{Legend}

Figure 1 Title: Individual items ranked by frequency with which they were used

Caption: The item numbers in this figure correlate with the rank numbers in Table 2, first column. 
Table 1: Outcome scoring systems used in the literature to determine outcome after treatment for calcaneal fracture.

\begin{tabular}{|c|c|c|c|}
\hline Outcome score & (First) author & Year & $\overline{\text { Citation }}$ \\
\hline Allan criteria $^{28}$ & Allan & 1955 & 2 \\
\hline Maxfield-McDermott criteria $^{29}$ & Maxfield & 1955 & 3 \\
\hline Lindsay-Dewar criteria $^{30}$ & Lindsay & 1958 & 2 \\
\hline Rowe outcome score ${ }^{16}$ & Rowe & 1963 & 7 \\
\hline Nade-Monahan questionnaire $^{31}$ & Nade & 1973 & 1 \\
\hline Mignot criteria $^{12}$ & Mignot & 1975 & 2 \\
\hline Fayt criteria ${ }^{32}$ & Fayt & 1978 & 1 \\
\hline Modified Mazur ankle score ${ }^{33}$ & Mazur & 1979 & 3 \\
\hline Noble-McQuillan criteria $^{34}$ & Noble & 1979 & 1 \\
\hline Grenoble score $^{35}$ & Champetier & 1979 & 1 \\
\hline Mestdagh score $^{36}$ & Mestdagh & 1984 & 2 \\
\hline Myerson scoring system $^{17}$ & Myerson & 1986 & 1 \\
\hline SO.F.C.O.T. criteria ${ }^{35}$ & Simon & 1988 & 4 \\
\hline Zwipp score 37 & Zwipp & 1989 & 9 \\
\hline Creighton-Nebraska $^{24}$ & Crosby & 1990 & 19 \\
\hline Bradley disability score ${ }^{38}$ & Bradley & 1990 & 1 \\
\hline Melcher-score ${ }^{39}$ & Melcher & 1991 & 2 \\
\hline Iowa Calcaneal Score ${ }^{26}$ & March & 1992 & 2 \\
\hline Buckley outcome score ${ }^{40}$ & Buckley & 1992 & 3 \\
\hline Maryland Foot Score $^{11}$ & Sanders & 1992 & 29 \\
\hline Parmar outcome criteria $^{41}$ & Parmar & 1993 & 1 \\
\hline Paley-Hall score ${ }^{42}$ & Paley & 1993 & 6 \\
\hline Letournel outcome criteria $^{43}$ & Letournel & 1993 & 1 \\
\hline Fernandez outcome criteria $^{44}$ & Fernandez & 1993 & 1 \\
\hline AOFAS hindfoot score ${ }^{23}$ & Kitaoka & 1994 & 51 \\
\hline Modified Merle D'Aubigné hip score ${ }^{45}$ & Kundel & 1996 & 10 \\
\hline Calcaneal fracture scoring system ${ }^{26}$ & Kerr & 1996 & 12 \\
\hline Functional Outcome Assessment ${ }^{46}$ & Thordarson & 1996 & 3 \\
\hline Musculoskeletal Functional Assessment ${ }^{47}$ & Martin & 1996 & 1 \\
\hline Oral Analogue Scale (OAS) ${ }^{48}$ & Morin & 1998 & 1 \\
\hline Strømsøe rating system ${ }^{49}$ & Strømsøe & 1998 & 1 \\
\hline Hannover Scoring System (HSS) ${ }^{50}$ & Thermann & 1999 & 2 \\
\hline Mod. Hannover Questionnaire (HQ) ${ }^{50}$ & Thermann & 1999 & 2 \\
\hline Kiel Calcaneus Score $^{51}$ & Schwall & 2000 & 1 \\
\hline
\end{tabular}

Overview of disease-specific outcome scores developed or used for the evaluation of calcaneal fractures, summarized by year of publication. The first author and citation frequency are mentioned. 
Table 2: Correlation between individual items and total outcome scores.

\begin{tabular}{|c|c|c|c|c|c|}
\hline & \multirow[t]{2}{*}{ Score items (Rank number) } & \multicolumn{3}{|c|}{ Total outcome score } & \multirow[t]{2}{*}{ VAS } \\
\hline & & AOFAS & MFS & $\mathbf{C N}$ & \\
\hline \multirow{16}{*}{ 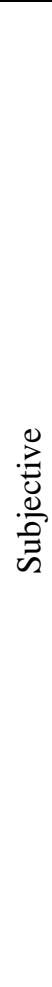 } & Pain (1) & $0.90 *$ & $0.77 *$ & $0.73 *$ & $0.61 *$ \\
\hline & Pain activity (8) & $0.90 *$ & $0.77 *$ & $0.73 *$ & $0.61 *$ \\
\hline & Pain rest (15) & $0.50 *$ & $0.37 *$ & $0.48^{*}$ & $0.31+$ \\
\hline & Activity limitations (11) & $0.83 *$ & $0.81 *$ & $0.70^{*}$ & $0.70 *$ \\
\hline & Walking distance (4) & $0.65 *$ & $0.54 *$ & $0.60 *$ & $0.54 *$ \\
\hline & Walking surface (9) & $0.60 *$ & $0.64 *$ & $0.57 *$ & $0.48 *$ \\
\hline & Gait abnormality (5) & $0.73 *$ & $0.77 *$ & $0.65^{*}$ & $0.58 *$ \\
\hline & Climb stairs (17) & ns & $0.34 t$ & $0.37 *$ & $0.39 *$ \\
\hline & Cosmesis (23) & $0.45^{*}$ & $0.55^{*}$ & $0.37 t$ & $0.34 *$ \\
\hline & Stability objectively (20) & ns & ns & ns & $\mathrm{ns}$ \\
\hline & Stability subjectively (21) & $0.52 *$ & $0.61 *$ & $0.58 *$ & $0.44 *$ \\
\hline & Shoe type (7) & $0.29 \ddagger$ & $0.51 *$ & $0.30 \ddagger$ & $\mathrm{ns}$ \\
\hline & Shoe size (22) & ns & ns & ns & ns \\
\hline & Support/aids (12) & $0.37 *$ & $0.40^{*}$ & $0.40 *$ & $0.38^{*}$ \\
\hline & Return to work (2) & $0.96 *$ & $0.56^{*}$ & $0.76^{*}$ & $0.60 *$ \\
\hline & Swelling (13) & $0.46^{*}$ & $0.65^{*}$ & $0.55^{*}$ & $0.62 *$ \\
\hline \multirow{7}{*}{ 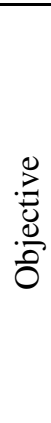 } & Calf circumference (19) & $\mathrm{ns}$ & ns & ns & ns \\
\hline & Walk on toes (10) & $0.45 \div$ & $0.42 \%$ & $0.36 t$ & $0.41 \%$ \\
\hline & Walk on heels (16) & $\mathrm{ns}$ & ns & ns & ns \\
\hline & Heel width (18) & $\mathrm{ns}$ & ns & ns & ns \\
\hline & Hindfoot motion (3) & $0.40 *$ & ns & $0.42 *$ & $0.36+$ \\
\hline & Sagittal motion (6) & ns & $\mathrm{ns}$ & ns & $\mathrm{ns}$ \\
\hline & Alignment (14) & $0.36 t$ & $0.30 \ddagger$ & $0.30 \ddagger$ & $0.36+$ \\
\hline
\end{tabular}

For item-total score correlations and item-VAS the Spearman rank correlation coefficient is presented. The rank numbers between brackets in the first column correspond with figure 1. VAS, Visual Analogue Scale for patient satisfaction; AOFAS, American Orthopaedic Foot and Ankle Society Hindfoot Score; MFS, Maryland Foot Score; CN, Creighton-Nebraska score; ns, not significant; $*$, significant at the $\mathrm{p}<0.001$ level; $\ddagger$, significant at the $\mathrm{p}<0.05$ level. 